(C) 2005 American Chemical Society, Anal. Chem., Rubio ac0502450 Supporting Info Page 1

\title{
Displacement Enzyme Linked Aptamer Assay
}

\section{(DELAA)}

Eva Baldrich ${ }^{a^{*}}$, Josep Lluis Acero ${ }^{a}$, Gunter Reekmans $^{c}$, Wim Laureyn ${ }^{c}$, and Ciara K. O'Sullivan ${ }^{a, b}$

${ }^{a}$ Department of Chemical Engineering, Universitat Rovira i Virgili, Avinguda Països Catalans 26, 43007 Tarragona, Spain.

b Institució Catalana de Recerca i Estudis Avançats (ICREA), Passeig Lluís Companys 23, 08010 Barcelona, Spain

${ }^{c}$ MCP-ART, Interuniversity Microelectronics Centre (IMEC), Kapeldreef 75, B-3001 Heverlee, Belgium

eva.baldrich@urv.net

* Corresponding Author: Eva Baldrich, eva.baldrich@urv.net Tel: 34-977-558740, Fax: 34-977-559667 


\section{MATERIAL AND METHODS}

\section{Surface Plasmon Resonance Elucidation of affinity constants}

The Biacore 2000 Surface Plasmon Resonance instrument was used at a constant temperature of $25^{\circ} \mathrm{C}$ and the autosampler racks were thermostatted at $4^{\circ} \mathrm{C}$ to prevent quick degradation of the thrombin.

Amine-modified TBA (TBA- $\mathrm{NH}_{2}$ ) was immobilized on a Biacore gold chip (SIA kit) via a mixed $S A M{ }^{1-3}$. The chip was rinsed with acetone and cleaned with a UV/Ozone treatment (15 min). After cleaning, the sample was immediately immersed for $3 \mathrm{~h}$ into a mixed solution of thiols (10\% (v/v) of 16-MHA ( $1 \mathrm{mM}$ in ethanol) and $90 \%(\mathrm{v} / \mathrm{v})$ of 11-MUOH (1mM in ethanol) to form a mixed SAM. Immobilization of the TBA was accomplished via the coupling of its amine group to the carboxylic group present on the mixed SAM. A continuous flow of 10mM HEPES pH 8.0 supplemented with $50 \mathrm{mM} \mathrm{KCl}(5 \mu \mathrm{l} / \mathrm{min})$ was maintained between the different injections during the immobilization protocol. The carboxylic groups were activated by addition of $50 \mu \mathrm{l}$ of a 1:1 mixture of EDC ( $400 \mathrm{mM}$ in water) and NHS (100mM in water). Then, $132 \mu 1$ of $0.5 \mu \mathrm{M}$ of the TBA- $\mathrm{NH}_{2}$ was immobilized using $10 \mathrm{mM}$ HEPES containing $0.6 \mathrm{mM}$ hexadecyltrimethylammonium bromide. This compound was added to block the negative charge of the aptamers, otherwise the pre-concentration effect would not occur. Finally, the surface was deactivated using $50 \mu 1$ of $1 \mathrm{M}$ ethanolamine solution $\mathrm{pH} 8.5$ followed by two $1 \mathrm{~min}$ pulses of a $2 \mathrm{M} \mathrm{NaCl}$ solution in order to homogenize the modified gold surface and to remove nonspecifically bound molecules from the surface. A random DNA sequence of equivalent length as the TBA, but which theoretically had no specificity towards thrombin was used as a control.

The study of the effect of modification of the thrombin on the ability of the TBA to bind it was carried out in 10mM HEPES pH 8.0 in the presence and in the absence of $50 \mathrm{mM} \mathrm{KCl}$ as running buffer. This study was performed for native and modified thrombin (i.e. labeled with horseradish peroxidase enzyme). Different dilutions of (modified) thrombin $(0,2.0,5.1,12.8,32,80$ and $200 \mathrm{nM})$ in running buffer were injected (at a flow rate of $5 \mu \mathrm{l} / \mathrm{min}$ for 10 minutes (association phase) and afterwards flushed with running buffer for 6 minutes (dissociation phase). For the regeneration of 
the TBA-coated surface two 1 minute pulses of a $2 \mathrm{M} \mathrm{NaCl}$ solution were used. Kinetic and affinity constants were calculated using the BIAevaluation 3.1 software.

\section{References:}

1. Frederix, F.; Bonroy, K.; Laureyn, W.; Reekmans, G.; Campitelli, A.; Dehaen, W.; Maes, G., Langmuir, 2003, 19, 4351-4357

2. Geukens, N., Frederix, F., Reekmans, G., Lammertyn, E., van Mellaert, L., Dehaen, W., Maes, G., Anné, J., Biochem Biophys Res Com, 2004, 314, 459

3. Huang, L., Saerens, D., Reekmans, G., Friedt, J.-M., Frederix, F., Francis, L. Muyldermans, S., Campitelli, A., Immuno Biosen Bioelec, 2005, in pres

\section{RESULTS:}

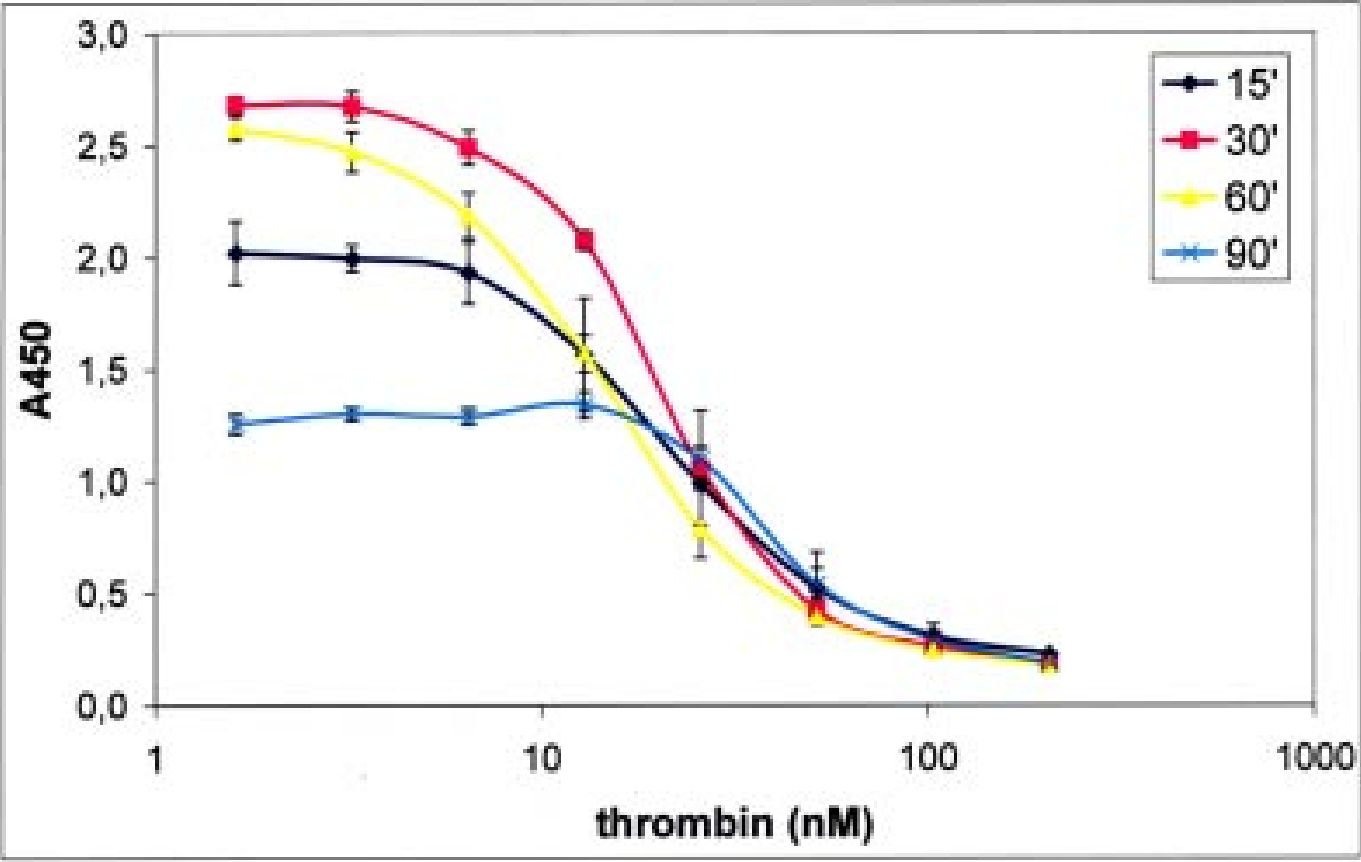

Figure A: Effect of incubation time on performance of competition assay.

Enzyme-labeled and unmodified thrombin were allowed to compete in solution during $10-90$ minutes at $4^{\circ} \mathrm{C}$ for the TBA previously immobilized on microtiter plates. 


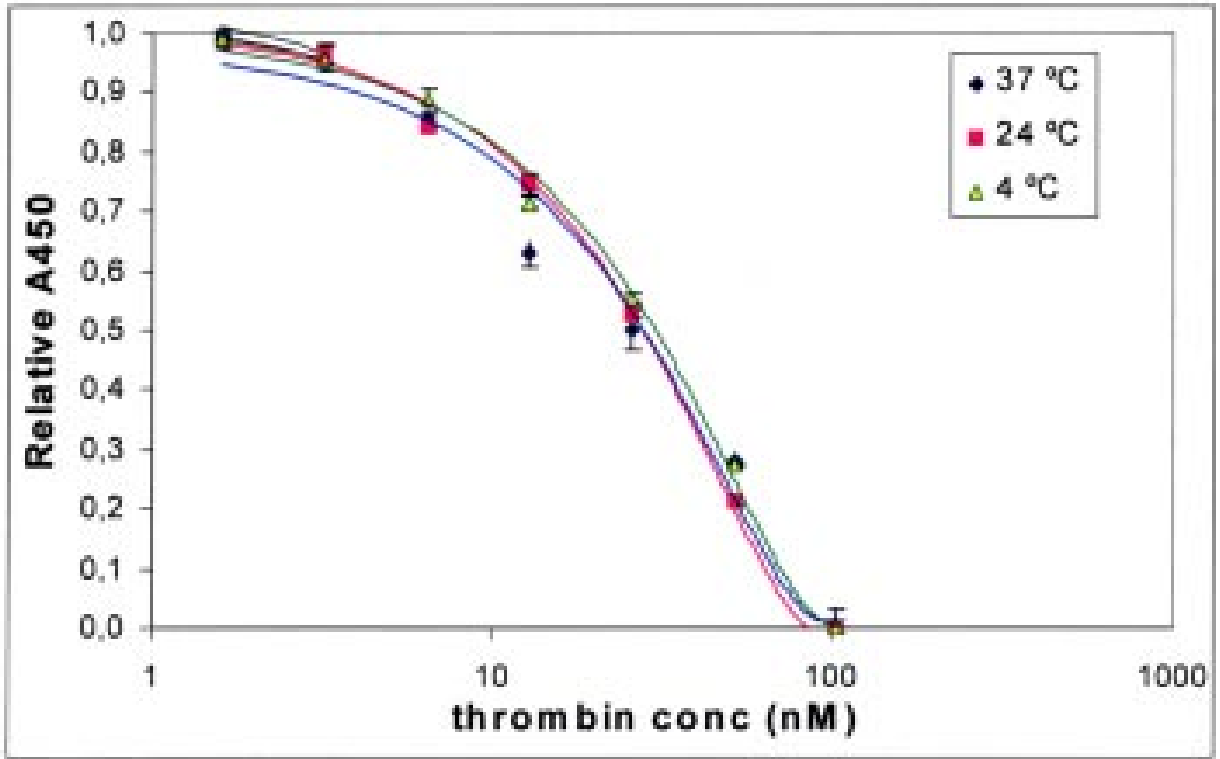

Figure B: Effect of the incubation temperature on the displacement ELAA efficiency. The displacement assay was carried out as described in the Material and Methods section, except that the displacement step was allowed to proceed for 30 minutes at 4,24 or $37^{\circ} \mathrm{C}$. The data were corrected by subtracting the background values and considering the maximal absorbance as 1 .

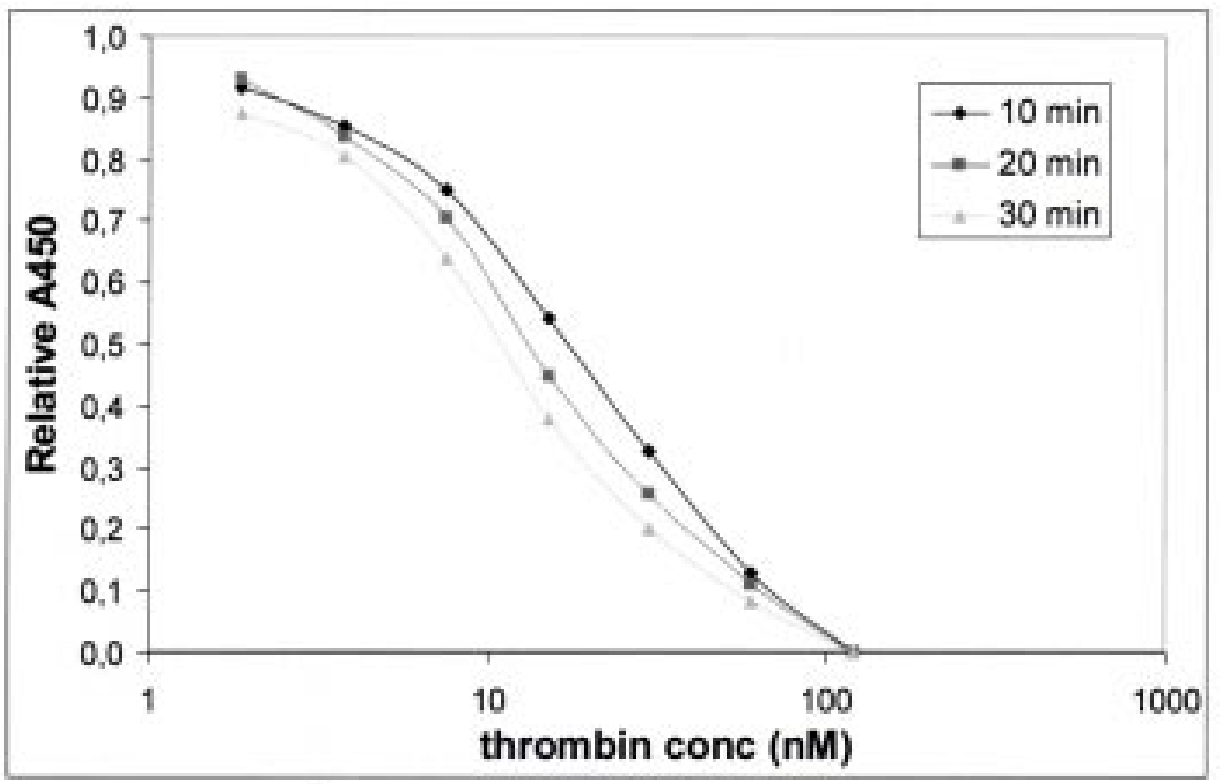

Figure C: Effect of the incubation time on the displacement ELAA efficiency.

The displacement assay was carried out as described in the Material and Methods section, except that the displacement step was allowed to proceed for 10,20 or 30 minutes at $4^{\circ} \mathrm{C}$. The data were corrected by subtracting the background values and considering the maximal absorbance as 1 . 